\title{
EXPERIÊNCIAS DE PROFISSIONAIS DE SAÚDE NO MANEJO DA VIOLÊNCIA INFANTIL
}

\author{
THE EXPERIENCES OF HEALTH PROFESSIONALS \\ WITH THE MANAGEMENT OF VIOLENCE AGAINST \\ CHILDREN
}

\section{EXPERIENCIAS DE PROFESIONALES DE LA SALUD EN EL MANEJO DE LA VIOLENCIA INFANTIL}

\author{
Leidiene Ferreira Santos ${ }^{1}$ \\ Ana Carolina Rodrigues de Sousa Javaé2 \\ Maraína Moreira da Costa ${ }^{2}$ \\ Maitê da Veiga Feitoza Borges Silva ${ }^{3}$ \\ Cintia Flôres Mutti ${ }^{4}$ \\ Leonora Rezende Pacheco5
}

Como citar este artigo: Santos LF, Javaé ACRS, Costa MM, Silva MVFB, Mutti CF, Pacheco LR. Experiências de profissionais de saúde no manejo da violência infantil. Rev baiana enferm. 2019;33:e33282.

\begin{abstract}
Objetivo: conhecer a experiência de profissionais de saúde da atenção básica no manejo da violência infantil. Método: estudo qualitativo, descritivo, exploratório. Entrevistaram-se profissionais de saúde que atuavam em uma Unidade de Saúde da Família. A coleta de dados ocorreu por meio de entrevistas norteadas por questões relacionadas à assistência à criança e à família em risco ou situação de violência. Submeteram-se os depoimentos à Análise de Conteúdo. Resultados: identificaram-se várias representações da violência na perspectiva dos entrevistados, as quais deram origem à categoria "Lidando com a violência contra a criança: fatores que comprometem a assistência". Conclusão: a experiência de profissionais de saúde da atenção básica no manejo da violência infantil experimenta diversas dificuldades na prática, especialmente relacionadas à falta de conhecimento, interferência e participação da família e desarticulação da rede de proteção infantil.
\end{abstract}

Descritores: Crianças. Defesa da Criança e do Adolescente. Maus-tratos Infantis. Violência. Violência Doméstica. Pessoal de Saúde.

Objective: getting to know the experience of primary healthcare professionals in the management of violence against children. Method: qualitative, descriptive, exploratory study. Health professionals who work in a Family Health Unit were interviewed. Data collection took place through interviews guided by questions regarding child assistance and families in risk of violence or situations of violence. Participant statements were submitted to Content Analysis. Results: many representations of violence from the perspective of interviewees were found, from which emerged the category "Dealing with violence against children: factors that compromise assistance". Conclusion: the experience of primary healthcare professionals in the management of child violence goes through many difficulties in practice, especially those related to lack of knowledge, interference and participation of the family, and to a disarticulated child protection network.

Descriptors: Child. Child Advocacy. Protection. Child Abuse. Violence. Domestic Violence. Health Personnel.

\footnotetext{
Enfermeira. Doutora em Ciências da Saúde. Professora da Universidade Federal de Tocantins. Palmas, Tocantins, Brasil. leidienesantos@uft.edu.br. https://orcid. org/0000-0002-2969-6203

Enfermeira. Pesquisadora independente. Palmas, Tocantins, Brasil. https://orcid.org/0000-000 I-5409-2500; https://orcid.org/0000-000 I-7030-4577

Enfermeira. Especialista em Enfermagem Obstétrica. Universidade Federal de Tocantins. Palmas, Tocantins, Brasil. https://orcid.org/0000-000 I-6I I7-8775

Enfermeira. Mestre em Enfermagem. Professora da Universidade Federal de Santa Maria, Santa Maria, Rio Grande do Sul, Brasil. https://orcid.org/0000-0003-0437-2568

Enfermeira. Doutora em Enfermagem. Professora da Universidade Federal de Goiás. Goiânia, Goiás, Brasil. https://orcid.org/0000-000 I-6048-39| I
} 
Objetivo: conocer la experiencia de profesionales de salud de la atención primaria en el manejo de la violencia infantil. Método: estudio cualitativo, descriptivo, exploratorio. Se entrevistó a los profesionales de salud de una Unidad de Salud de la Familia. Se colectó los datos por medio de una entrevista guiada por cuestiones relacionadas a la asistencia a los niños y a las familias bajo riesgo o situaciones de violencia. Se sometió sus declaraciones al Análisis de Contenido. Resultados: se identificó numerosas representaciones de la violencia desde la perspectiva de los entrevistados, que originaron la categoria "Manejando la violencia contra niños: factores que comprometen la asistencia". Conclusión: la experiencia de profesionales de salud de la atención primaria en el manejo de violencia infantil tiene muchas dificultades en la práctica, especialmente relacionadas a la falta de conocimiento, interferencia y participación de la familia, y a la desarticulación de la red de protección infantil.

Descriptores: Niños. Defensa del Niño. Maltrato a los Niños. Violencia. Violencia Doméstica. Personal de Salud.

\section{Introdução}

Sabe-se que situações de violência ocorrem em diferentes contextos e cenários e representam grave problema de saúde pública, especialmente por provocarem expressivo número de óbitos e morbidades. Cotidianamente, mulheres, crianças, adolescentes e idosos precisam conviver com abusos físico, sexual e psicológico, o que concorre para a ocorrência de transtornos biopsicossociais para essas pessoas e a sociedade ${ }^{(1)}$.

A violência infantil, por sua vez, apresenta-se como realidade alarmante que contribui para prejuízos físicos e emocionais durante a infância e, consequentemente, para a vida adulta ${ }^{(2-3)}$. Ela é conceituada como qualquer ação ou omissão dos pais, familiares, pessoas responsáveis, estabelecimentos públicos e privados e a sociedade, que provoque prejuízo às crianças ${ }^{(4)}$.

Somente em 2011 foram registrados, no Brasil, 17.900 casos de violência contra crianças de até nove anos, sendo 33\% naquelas com até um ano, 35,8\% nas de dois a cinco anos e 31,2\% nas de seis a nove anos ${ }^{(5)}$. Destaca-se que os pais aparecem como principais perpetradores da violência, sobretudo em crianças na faixa etária menor ou igual a cinco anos, e a negligência apresenta-se como a primeira forma de maus-tratos, constituindo-se como problema de ordem social grave que não tem visibilidade na sociedade ${ }^{(6)}$.

Segundo sua natureza, a violência infantil pode ser classificada em física (ato violento com uso da força física de maneira intencional, não acidental, que pode ferir, lesar, provocar dor e sofrimento ou destruir a pessoa), psicológica (toda ação que exponha a risco ou cause dano à autoestima, à identidade ou ao desenvolvimento da criança), sexual (ato ou jogo com intenção de estimular sexualmente a criança, em que os autores estejam em estágio de maturidade psicossexual mais adiantado que a criança) e negligência (omissões dos adultos, ao deixarem de prover as necessidades básicas para o desenvolvimento da criança) ${ }^{(4)}$.

Salienta-se que nenhum tipo de violência é justificável e toda aquela contra crianças pode ser prevenida ${ }^{(6)}$. Requerem-se, entretanto, para a desconstrução desse fenômeno culturalmente aceito, conscientização e comprometimento de toda a sociedade, especialmente frente às iniquidades do mundo contemporâneo ${ }^{(5)}$.

No Brasil, está regulamentada como mecanismo de proteção à criança, a obrigatoriedade de notificação compulsória dos casos suspeitos ou confirmados de agressão infantil, por profissionais de saúde, professores ou responsáveis pelo estabelecimento de atenção à saúde e de ensino fundamental, pré-escola ou creche. Configura-se infração administrativa, sujeita a multa de três a vinte salários de referência, a não comunicação de tais eventos ao Conselho Tutelar da respectiva localidade de moradia da vítima ou demais órgãos competentes ${ }^{(7)}$. As fichas de notificação representam ferramenta indispensável para a identificação e o manejo dos $\operatorname{casos}^{(8)}$.

Nessa perspectiva, destaca-se o papel dos profissionais que atuam na Estratégia Saúde da Família (ESF), para o registro adequado 
das agressões, contribuindo para a promoção e a proteção da criança ${ }^{(9-10)}$. Na ESF, é possível realizar acompanhamento e avaliação integrais e sistematizados, durante o crescimento e desenvolvimento da criança, bem como identificar problemas aos quais ela possa estar exposta no contexto em que vive ${ }^{(11)}$.

Está evidente, entretanto, que os profissionais de saúde apresentam limitações em relação ao reconhecimento e manejo da violência, além de dificuldades para uma abordagem integrada e intersetorial $^{(10)}$. Apresentam-se ainda, em muitas instituições, tais como as de saúde e educação, baixas taxas de denúncias ${ }^{(12)}$, ainda que falhas nesses aspectos comprometam a prevenção e o enfrentamento da violência infantil ${ }^{(5,12)}$.

Apesar de os profissionais de saúde desempenharem importante papel na rede de proteção da criança e no manejo dos casos de violência ${ }^{(12-13)}$, e de os direitos ligados à infância e à juventude estarem expressos em constituições e declarações nacionais $^{(4,7)}$ e internacionais ${ }^{(14)}$, a universalização da garantia desses direitos ainda é um desafio atual ${ }^{(5)}$.

Assim, acredita-se que os resultados desta pesquisa podem contribuir para o direcionamento de intervenções que atendam efetivamente às demandas de crianças e das famílias em risco ou situação de violência, qualificando e humanizando a assistência oferecida, bem como colaborar para a prevenção e a identificação dos casos, de modo a interromper o ciclo e evitar reincidências.

O objetivo deste artigo é conhecer a experiência de profissionais de saúde da atenção básica no manejo da violência infantil.

\section{Método}

Trata-se de uma pesquisa de abordagem qualitativa, descritiva e exploratória, conduzida segundo as diretrizes do Confidence in Evidence of Reviews of Qualitative Research (ENTREQ) guidelines. Foram entrevistados profissionais que integravam duas equipes de uma Unidade de Saúde da Família (USF) localizada em Palmas, Tocantins, Brasil.
O município possuía 32 USF instaladas no perímetro urbano, que atendiam uma população estimada de aproximadamente 300 mil habitantes e executavam ações de prevenção e promoção da saúde.

A USF desta pesquisa possuía 3.629 famílias cadastradas e estava localizada numa região em que a população apresentava, em sua maioria, baixa renda. Integravam o quadro de profissionais que atuavam nas duas equipes de saúde da família, dois médicos clínico geral, duas enfermeiras, quatro técnicos de enfermagem, um odontólogo e nove agentes comunitários de saúde, totalizando 18 profissionais de saúde.

Optou-se pela referida unidade por se tratar de cenário de atividades práticas de alunos do Curso de Enfermagem da Universidade Federal do Tocantins. Poder-se-ão desenvolver, nesse sentido, com base nos resultados encontrados nesta pesquisa, intervenções com vistas a melhorar a assistência às crianças e famílias em risco ou situação de violência.

As entrevistas foram realizadas por uma pesquisadora treinada para essa finalidade e aconteceram nas dependências da unidade de saúde, nos meses de setembro de 2015 a janeiro de 2016, em uma sala de reuniões ou consultório dos participantes, de modo a garantir a privacidade necessária para a coleta de dados. Agendaram-se previamente as entrevistas, que foram gravadas em mídia digital e norteadas pelas seguintes questões: "Fale-me sobre suas experiências profissionais nos casos suspeitos ou confirmados de violência contra criança" e "Caso existam, fale-me sobre fatores que dificultam e facilitam sua atuação nos casos suspeitos ou confirmados de violência contra criança”.

As entrevistas foram transcritas pela pesquisadora que as realizou, e os dados foram submetidos à Análise de Conteúdo. Realizou-se, para tanto, leitura exaustiva e compreensiva do material, e buscou-se sistematizar as ideias iniciais que, posteriormente, foram agregadas em unidades que permitiram a descrição dos conteúdos apreendidos, com a elaboração de textos de acordo com a análise dos conteúdos e formação 
de categorias $^{(15)}$. Além da entrevistadora, mais três pesquisadoras participaram dessa etapa.

Utilizou-se como critério para a inclusão nesta pesquisa: ser profissional de saúde (médicos, enfermeiros, auxiliares e técnicos de Enfermagem, agentes comunitários de saúde e dentistas) que atuasse na USF há, pelo menos, seis meses, excluindo-se os profissionais afastados das atividades laborais por motivo de licença ou férias.

Atribuíram-se, a fim de preservar a identidade e evitar a exposição e o constrangimento dos entrevistados, para a apresentação dos resultados, letras, que representam os profissionais de saúde, e números. No sistema alfanumérico empregado, a letra "E" indica enfermeiro; "M", médico; "ACS", agente comunitário de saúde; e "TE", técnicos de Enfermagem. O número que segue estas letras corresponde à ordem de realização das entrevistas.

Esta pesquisa foi aprovada pela Secretaria Municipal de Saúde de Palmas, sob o protocolo 008-02/2015, e pelo Comitê e Ética em Pesquisa com seres humanos, sob o protocolo 086/2015, atendendo-se aos preceitos éticos da Resolução n. 466/2012 do Conselho Nacional de Saúde.

\section{Resultados}

Participaram desta pesquisa, no total, 13 profissionais de saúde, sendo 11 do sexo feminino e 2 do sexo masculino; 1 médico, 2 enfermeiros, 4 técnicos de Enfermagem e 6 agentes comunitários de saúde. O tempo de atuação na USF variou de 2 a 14 anos, e a faixa etária de 33 a 54 anos. Informa-se que um médico, um dentista e dois agentes comunitários de saúde não participaram por estarem em período de férias, e um agente comunitário recusou-se.

Nos depoimentos dos profissionais de saúde, identificaram-se várias vivências frente às situações de violência confirmadas ou suspeitas, as quais deram origem à categoria "Lidando com a violência contra a criança: fatores que comprometem a assistência” e subcategorias, que estão apresentadas no Quadro 1.

Quadro 1 - Lidando com a violência contra a criança: fatores que comprometem a assistência Categoria e subcategorias

(continua)

\begin{tabular}{|c|c|}
\hline Subcategorias & Depoimentos \\
\hline $\begin{array}{l}\text { Compreensão sobre } \\
\text { a violência }\end{array}$ & $\begin{array}{l}\text { Violência é atingir a integridade física da criança [...] Essa criança vai crescer uma crian- } \\
\text { ça com trauma. (ACS3). } \\
\text { Violência é tudo que deixa hematoma. (ACS4). } \\
\text { [...] se for uma agressão física [...] já é visivel [...] Se for uma agressão tipo sexual [...] que } \\
\text { acontece muito [...] aí já é mais, é mais complicado pra gente. (TE1). } \\
\text { [...] violência fïsica é fácil você ver, geralmente, bem fácil de identificar. A gente tem só } \\
\text { que tentar diferenciar os mecanismos da lesão. Agora, psicológico, depende muito do grau } \\
\text { da agressão psicológica. (M1). }\end{array}$ \\
\hline Interferência da Família & $\begin{array}{l}\text { [...] eu acho que a dificuldade vem da própria família. Às vezes, o ato de esconder, o ato } \\
\text { de não falar. Eu acho que essa é a dificuldade. (ACS2). } \\
\text { [...] a família esconde. Muitas vezes, tem o sigilo da família. Às vezes, a gente fica sabendo } \\
\text { por terceiros. (E1). } \\
\text { [...] às vezes, é a forma que os pais, assim, ou então o próprio agressor [...] interfere, enten- } \\
\text { deu? A gente perguntar pra criança ou então a gente tentar conversar com ela, o pai não } \\
\text { deixa. (TE3). }\end{array}$ \\
\hline Fragilidade na Rede & $\begin{array}{l}\text { [...] então, nós temos essa função que, muitas vezes, é falha, devido ao sistema, porque as } \\
\text { pessoas sofrem a violência institucional até mesmo no sistema, porque não funciona. E aí } \\
\text { nós também sofremos. (ACS2). } \\
\text { [...] outra dificuldade também é a questão da gente tentar localizar essa assistência que, } \\
\text { às vezes, tem toda uma burocracia. (TE3). } \\
\text { Falta integrar, porque a saúde tá aqui, o conselho tá aqui. Tem que integrar esses polos. } \\
\text { (E2). }\end{array}$ \\
\hline
\end{tabular}


Quadro 1 - Lidando com a violência contra a criança: fatores que comprometem a assistência Categoria e subcategorias

\begin{tabular}{|l|l|}
\hline Subcategorias & \multicolumn{1}{c|}{ Depoimentos } \\
\hline Omissão do cuidado & $\begin{array}{l}\text { [...] eu não vou denunciar, a não ser que seja muito espancamento. (ACS5). } \\
\text { Mesmo, às vezes, a gente vê acontecendo, mais fica "o filho não é meu, não é meu parente, } \\
\text { eu não conheço". Então, eu prefiro ficar calada. (TE2). } \\
{[\ldots .] \text { a única coisa que você pode fazer é o que tá no teu alcance, no caso, que seria passar }} \\
\text { pelo médico [...] porque, na realidade, a gente mesmo não tem como a gente fazer nada. } \\
\text { (TE4). }\end{array}$ \\
\hline Medo de Retaliação & $\begin{array}{l}\text { [...] então, assim, eu posso ver a agressão, mas, às vezes, fico com medo de tá passando pra } \\
\text { frente, porque eu tenho que entrar lá novamente. (ACS1). } \\
{[\ldots] \text { a gente tem medo, por exemplo, de eu ir lá na casa e ver que foi agredido e dali sair }} \\
\text { uma denúncia, e eles lfamília] acharem que foi eu, e eu ser agredida depois. (ACS5). } \\
{[\ldots . .] \text { é um assunto muito complicado, porque envolve muita gente, e a gente tem medo, }} \\
\text { a gente tem receio. Não só eu, mas a equipe toda. (E2). }\end{array}$ \\
\hline
\end{tabular}

Fonte: Elaboração própria.

\section{Discussão}

Verificou-se, nesta pesquisa, que os profissionais de saúde têm compreensão limitada em relação ao conceito de violência infantil e dificuldades para reconhecer, na prática, sua classificação e formas de expressão. De modo semelhante, estudo realizado com 877 profissionais de saúde, na China, evidenciou conhecimento insuficiente na identificação de possíveis casos de maus-tratos infantis, especialmente o abuso sexual, e que apenas 3,19\% dos entrevistados haviam recebido treinamento sobre o manejo da violência infantil ${ }^{(16)}$.

Detalha-se que alguns tipos de violência, tais como o trabalho infantil e o abandono, sequer foram mencionados pelos participantes desta pesquisa. Além disso, muitos limitaram violência às ocorrências de lesões físicas. Tais fatos corroboram falhas na proteção eficaz das crianças $^{(16-17)}$.

Acredita-se que fragilidades na formação de recursos humanos na área da saúde sobre maus-tratos que acometem grupos vulneráveis, incluindo crianças, contribuam para o exposto. Sabe-se que essa temática não é contemplada na maioria das matrizes curriculares dos cursos de ensino superior da saúde, incluindo a pós-graduação, e não se apresenta como alvo de capacitação na formação continuada das equipes da estratégia saúde da família ${ }^{(18)}$.

Vê-se, nesse sentido, como importante a realização de estratégias de formação continuada, bem como ampliar mecanismos de suporte às equipes de saúde, a fim de instrumentalizá-las para atuarem efetivamente na proteção infantil ${ }^{(17,19)}$.

Apresenta-se também como entrave para a identificação e a atuação da equipe de saúde no enfrentamento da violência contra a criança, segundo relatos dos profissionais entrevistados, a participação familiar nas agressões. Estudos nacionais ${ }^{(5,19-20)}$ e internacionais ${ }^{(21-22)}$ evidenciam a família como principal perpetradora de abuso infantil.

Nota-se, assim, que nem sempre o núcleo familiar cumpre o papel de proteger a criança. Ao contrário, abnega-se de suas responsabilidades e, até mesmo, torna-se executor de atos de agressão que perturbam o desenvolvimento emocional, comprometendo o desempenho psicossocial e o bem-estar da criança ${ }^{(23)}$.

Mudar essa realidade configura-se como desafio atual e complexo e requer romper com o paradigma de que maus-tratos infantis sejam ações naturais ou apenas um modo particular de os pais lidarem com seus filhos. Para tanto, é indispensável, às famílias e à sociedade, que a violência seja entendida como uma forma 
grave de desrespeito aos direitos humanos fundamentais ${ }^{(19-20)}$.

Necessita-se de medidas públicas voltadas ao esclarecimento de toda a sociedade em relação às inúmeras formas de expressão da violência e suas consequências, objetivando mudar padrões culturalmente enraizados, nos quais as ações e os atos violentos da família são frequente e erroneamente implementados como mecanismos para a educação da criança ${ }^{(20)}$.

Nesse cenário, percebe-se que, ao mesmo tempo em que a proximidade das famílias contribui para que os profissionais que atuam na atenção primária identifiquem demandas reais da comunidade, propiciando assistência singular e integral, também os expõe ao risco de conflitos e represálias. Do mesmo modo, a falta de mecanismos de proteção contribui para a negação e a omissão do papel que deveriam exercer na rede de proteção à criança.

É importante pontuar que o medo de sofrer algum tipo de agressão aflige os profissionais em sua prática cotidiana e faz com que, muitas vezes, exerçam assistência reducionista, omitindo-se do papel que deveriam cumprir.

O temor de ser identificado e sofrer represálias, por parte dos agressores, especialmente pela carência de proteção ao profissional que realiza a denúncia e pelas fragilidades na rede de cuidados, configura-se barreira para a implementação de uma conduta eficaz nos casos de violência contra a criança. Expressa-se, por esse quadro, a necessidade de que sejam revistos aspectos legais e a organização dos serviços, de maneira a resguardar os profissionais notificantes ${ }^{(20)}$, as famílias e as crianças violentadas.

É preciso também considerar as demandas de cuidado dos profissionais de saúde que atuam em situações complexas, tais como casos de violência infantil. Identificar os sentimentos vivenciados por esses atores pode contribuir para que sejam implementadas ações que reestabeleçam e promovam sua saúde ${ }^{(24)}$. Estratégias que os acolham, encorajem e enfatizem a importância do trabalho que realizam podem colaborar para melhorar suas habilidades de lidar com os desafios associados ao abuso infantil e promover autonomia, segurança e apoio emocional ${ }^{(25)}$.

É válido pontuar que o profissional, ao não se sentir acolhido, protegido e/ou não compreender a importância do papel que deve desempenhar para a proteção da criança, pode realizar assistência fragmentada, desumana e omissa, alicerçada no modelo biomédico de atenção, limitada a identificar e a tratar doenças, como foi observado neste e em outros estudos ${ }^{(8,16-17)}$.

Com isso, presentifica-se a assistência reducionista, em que o foco é unicamente o tratamento do corpo físico, negligenciando-se as demandas biopsicossociais de crianças e famílias, no agir de muitos profissionais de saúde. Esse tipo de comportamento não colabora para o enfrentamento da violência infantil, pois, para tanto, são necessárias as atitudes de empatia, comprometimento, responsabilização, envolvimento e participação social. Contribui-se, por meio dessa conduta, para que não sejam realizadas ações preventivas e protetivas, bem como haja a transferência da responsabilidade de atuar nos casos de violência para outras instituições ${ }^{(8)}$.

A assistência é desqualificada pelas ações reducionistas, colaborando para que sejam negligenciadas demandas biopsicossociais dos usuários dos serviços de saúde, não permitindo a cada um ser percebido em sua totalidade e individualidade. Tornam-se imprescindíveis, desse modo, ações de educação continuada que instrumentalizem os profissionais de saúde para implementarem a assistência integral e humanizada e para melhor conhecimento de como deve ser o funcionamento da rede de assistência à criança para o enfrentamento da violência, com ênfase nas responsabilidades individuais e coletivas ${ }^{(9)}$.

Nesta pesquisa, também se identificou que os serviços de assistência à criança ainda trabalham de modo desarticulado e assistemático, prejudicando a consolidação de uma rede que atue efetivamente no enfrentamento da violência. Apontaram-se falhas na comunicação, lentidão, mau funcionamento dos serviços e burocracias, vistos como barreiras para se estabelecer e integrar os serviços de proteção à criança, contribuindo para 
que o profissional de saúde não desempenhasse, de modo satisfatório, o seu papel na rede.

Acredita-se que o funcionamento de "Redes Institucionais" que integram serviços de assistência configura-se em estratégia eficaz para a prevenção e a interrupção do ciclo da violência $^{(9,20)}$, por apresentar mecanismos protetivos da criança e também do profissional de saúde. Todavia, há inúmeras dificuldades para se estabelecer e integrar as instituições de proteção à criança ${ }^{(10,20)}$. Tornam-se necessários, para superar essas limitações, a articulação de vontades, o diálogo permanente entre os atores, a busca de parceiros e a capacitação permanente para o trabalho coletivo e intersetorial, além de investimento financeiro ${ }^{(9)}$.

A capacitação dos profissionais de saúde para integrar o atendimento, viabilizar a identificação precoce, realizar a notificação dos casos e a efetivação do acompanhamento às crianças vítimas de violência e seus familiares representa um pilar para melhorar os indicadores da violência infantil $^{(8)}$. Assim, é cogente maior conscientização, tanto da população quanto de outros atores envolvidos no atendimento infanto-juvenil, sobre a importância da notificação desse agravo, bem como investimentos, por parte do poder público, em estratégias que contribuam para o avanço no enfrentamento da violência contra a criança ${ }^{(20)}$.

Nessa perspectiva, destacam-se os profissionais de saúde como atores fundamentais para implementar estratégias capazes de mudar os indicadores de violência ${ }^{(21,25)}$, tais como atividades educativas e atuação na prevenção e no tratamento na perspectiva da interdisciplinaridade, multiprofissionalidade, intersetorialidade e socialmente engajada, de modo a empoderar famílias e sociedade em relação ao papel que devem assumir para a proteção da criança, auxiliando na redução dos abusos infantis em suas distintas formas de expressão.

Apresenta-se como limitação, não obstante a relevância dos resultados apresentados nesta pesquisa, o fato de as entrevistas incluírem profissionais da Estratégia Saúde da Família, não sendo possível, desse modo, apresentar experiências ocorridas em outros setores que compõem a rede de proteção à criança.

\section{Conclusão}

Os resultados deste estudo deram visibilidade às diversas experiências vividas na prática das equipes de saúde que assistem crianças e famílias em risco ou situação de violência, tais como as limitações em relação a conhecer o conceito e os tipos de violência contra a criança, a naturalização da violência no ambiente familiar, a falta de apoio das famílias e da comunidade, a desarticulação entre as instituições que deveriam integrar a rede de proteção à criança, a assistência em saúde alicerçada no modelo reducionista, a falta de responsabilização e o medo de atuar nos casos. Tais dificuldades não estão restritas a uma classe profissional, sendo expressas por médicos, enfermeiros, técnicos de Enfermagem e agentes comunitários de saúde.

Identificou-se que as ações dos profissionais que atuam na UBS são, muitas vezes, insuficientes para a prevenção e o manejo da violência contra a criança, o que pode estar relacionado à complexidade do problema e à falta de conhecimentos e habilidades para a atuação efetiva. Tornam-se, assim, urgentes ações governamentais que garantam a qualificação aos profissionais de saúde que trabalham na ESF, para que possam atuar de maneira resolutiva, conforme preconiza o Estatuto da Criança e do Adolescente.

De modo geral, identificou-se que, na prática, os profissionais de saúde experienciaram falta de conhecimentos sobre violência infantil, conflitos com as famílias e mau funcionamento da rede de proteção à criança.

Acredita-se que está evidente a necessidade de ações capazes de empoderar as equipes de saúde em relação à importância do papel que devem assumir na rede de proteção à criança, haja vista que, sem a participação efetiva dos setores que integram essa rede, sempre haverá lacunas que refletirão em uma assistência fragmentada e pouco resolutiva. Desse modo, entende-se que os profissionais devem atuar como agentes sociais de mudanças engajados 
e politicamente conscientes dos seus direitos e deveres.

\section{Colaborações:}

1 - concepção, projeto, análise e interpretação dos dados: Leidiene Ferreira Santos, Ana Carolina Rodrigues de Sousa Javaé, Maraína Moreira da Costa e Maitê da Veiga Feitoza Borges Silva;

2 - redação do artigo e revisão crítica relevante do conteúdo intelectual: Leidiene Ferreira Santos, Cintia Flôres Mutti e Leonora Rezende Pacheco;

3 - aprovação final da versão a ser publicada: Leidiene Ferreira Santos, Cintia Flôres Mutti e Leonora Rezende Pacheco.

\section{Referências}

1. World Health Organization. Violence and injury prevention. Global status report on violence prevention 2014 [Internet]. Geneva; 2014 [cited 2019 Oct 22]. Available from: https://www. who.int/violence_injury_prevention/violence/ status_report/2014/en/

2. Nemeroff CB. Paradise lost: the neurobiological and clinical consequences of child abuse and neglect. Neuron. 2016 Mar;89(5):892-909. DOI: 10.1016/j.neuron.2016.01.019

3. Villodas MT, Litrownik AJ, Newton RR, Davis IP. Long-term placement trajectories of children who were maltreated and entered the child welfare system at an early age: consequences for physical and behavioral well-being. J Pediatr Psychol. 2016 Jan/Feb;41(1):46-54. DOI: 10.1093/jpepsy/jsv031

4. Brasil. Ministério da Saúde. Política Nacional de Redução da Morbimortalidade por Acidentes e Violências [Internet]. Brasília; 2001 [cited 2019 Oct 22]. Available from: http://bvsms.saude.gov. br/bvs/publicacoes/acidentes.pdf

5. Rates SMM, Melo EM, Mascarenhas MDM, Malta DC. Violence against children: an analysis of mandatory reporting of violence, Brazil 2011. Ciênc Saúde Colet. 2015 Mar;20(3):655-65. DOI: http://dx.doi. org/10.1590/1413-81232015203.15242014

6. Malta DC, Bernal RTI, Teixeira BSM, Silva MMA, Freitas MIF. Factors associated with violence against children in sentinel urgent and emergency care centers in Brazilian capitals. Ciênc Saúde Colet. 2017 Sep;22(9):2889-98. DOI: http://dx.doi. org/10.1590/1413-81232017229.12752017

7. Brasil. Lei n. 8.069, de 13 de julho de 1990. Dispõe sobre o Estatuto da Criança e do Adolescente e dá outras providências [Internet]. Brasília; 1990 [cited 2019 Oct 22]. Available from: http://www.planalto. gov.br/ccivil_03/LEIS/L8069.htm

8. Schek G, Silva MRS, Lacharité C, Cézar-Vaz MR, Bueno MEN, Ventura J. Professional practices that silence domestic violence against children and adolescents. Texto contexto-enferm. 2018 Mar;27(1):e1680016. DOI: http://dx.doi. org/10.1590/0104-07072018001680016

9. Egry EY, Apostólico MR, Morais TCP. Reporting child violence, health care flows and work process of primary health care professionals. Ciênc Saúde Colet. 2018 Jan;23(1):83-92. DOI: http://dx.doi. org/10.1590/1413-81232018231.22062017

10. Silva PA, Lunardi VL, Ribeiro JP, Oliveira AMN, Vasquez TCS. Notification of domestic violence against children and teens by health professionals in Brazil. Av Enferm. 2015 Jan/Apr;33(1):14250. DOI: http://dx.doi.org/10.15446/av.enferm. v33n1.40585

11. Yakuwa MS, Sartori MCS, Mello DF, Duarte MTC, Tonete VLP. Child Health Surveillance: nurses perspective. Rev Bras Enferm. 2015 May/June;68(3):330-6. DOI: http://dx.doi. org/10.1590/0034-7167.2015680302i

12. Moreira TNF, Martins CL, Feuerwerker LCM, Schraiber LB. The foundation of care: Family Health Program teams dealing with domestic violence situations. Saúde Soc. 2014 Jul/ Sep;23(3):814-27. DOI: http://dx.doi.org/10.1590/ S0104-12902014000300007

13. Janßen K, Greif D, Rothschild MA, Banaschak S. Relevance of medical reports in criminal investigations of cases of suspected child abuse. Int J Legal Med. 2017 Jul;131(4):1055-9. DOI: https://doi.org/10.1007/s00414-017-1533-6

14. World Health Organization. World report on violence and health: summary [Internet]. Geneva; 2002 [cited 2019 Oct 22]. Available from: http:// www.who.int/violence_injury_prevention/ violence/world_report/en/summary_en.pdf

15. Bardin L. Análise de conteúdo. 6a ed. Lisboa: Edições 70; 2011.

16. Li X, Yue Q, Wang S, Wang H, Jiang J, Gong L, et al. Knowledge, attitudes, and behaviours of healthcare 
professionals regarding child maltreatment in China. Child Care Health Dev. 2017 Nov;43(6):86975. DOI: https://doi.org/10.1111/cch.12503

17. Berthold O, Hoffmann U, Clemens V, Witt A, Fegert JM. Kinderschutz im Gesundheitswesen verbessern: Fachberatung, Weiterbildung und Forschung am Beispiel des misshandlungsbedingten Kopftraumas. Bundesgesundheitsblatt - Gesundheitsforschung - Gesundheitsschutz. 2019 Aug;62(8):960-9. DOI: https://dx.doi.org/10.1007/s00103-019-02982-2

18. Moreira CAR, Vieira LJES, Deslandes SF, Pordeus MAJ, Gama IS, Brilhante AVM. Factors associated with the report and adolescent abuse in primary healthcare Ciênc Saúde Colet. 2014 Oct;19(10):4267-76. DOI: http://dx.doi. org/10.1590/1413-812320141910.17052013

19. Nunes AJ, Sales MCV. Violência contra crianças no cenário brasileiro. Ciênc Saúde Colet. 2016 Mar;21(3):871-80. DOI: http://dx.doi. org/10.1590/1413-81232015213.08182014

20. Santos LF, Costa MM, Javae ACRS, Mutti CF, Pacheco LR. Factors that interfere with the confrontation of child violence by guardianship counselors. Saúde debate. 2019 Jan/Mar;43(120):137-49. DOI: http:// dx.doi.org/10.1590/0103-1104201912010

21. Chan KL. Child Victimization in the Context of Family Violence. Int $\mathrm{J}$ Environ Res Public
Health. 2019 Sep;16(19):3569. DOI: https://doi. org/10.3390/ijerph16193569

22. Nakaya N. Mother's attributions regarding children's behavior and maltreatment. Shinrigaku Kenkyu. 2016 Apr;87(1):40-9. PMID: 27180512

23. Monteiro ACS, Fernandes ATRS, Oliveira ABM, Peixoto IVP, Pamplona MCCA. Mothers' perspective on violence against children: constructing meanings. Rev Bras Enferm. 2018 Jan/Feb;71(1):34-9. DOI: http://dx.doi.org/10.1590/0034-7167-2016-0568

24. Schek G, Silva MRSS. Feelings Experienced by Professionals Who Work in Services to Protect Children and Adolescents Victims of Intrafamily Violence and its Effects in Daily Practice. J res: fundam care. Online. 2018 Jul/Sep;10(3):764-9. DOI: http://dx.doi.org/10.9789/2175-5361.2018. v10i3.764-769

25. Albaek AU, Binder PE, Milde AM. Entering an emotional minefield: professionals' experiences with facilitators to address abuse in child interviews. BMC Health Serv Res. 2019 May;19(1):1-12. DOI: https://doi.org/10.1186/s12913-019-4128-8

Recebido: 26 de agosto de 2019

Aprovado: 29 de outubro de 2019

Publicado: 18 de dezembro de 2019

A Revista Baiana de Enfermagem utiliza a Licença Creative Commons - Atribuição-NãoComercial 4.0 Internacional. https://creativecommons.org/licenses/by-nc/4.0/

Este artigo é de acesso aberto distribuído sob os termos da Licença Creative Commons (CC BY-NC). Esta licença permite que outros remixem, adaptem e criem a partir do seu trabalho para fins não comerciais. Embora os novos trabalhos tenham de lhe atribuir o devido crédito e não possam ser usados para fins comerciais, os usuários não têm de licenciar esses trabalhos derivados sob os mesmos termos. 